Ibn Al-Haitham Jour. for Pure \& Appl. Sci. 34 (2) 2021

Ibn Al Haitham Journal for Pure and Applied Science

Journal homepage: http://jih.uobaghdad.edu.iq/index.php/j/index

\title{
Solving Fuzzy Attribute Quality Control Charts with proposed Ranking Function
}

\author{
Esraa Dhafer Thamer \\ Israadafer60@gmail.com
}

\author{
Iden Hasan Hussein \\ Idenalkanani@yahoo.com
}

Department of Mathematics, College of Science for woman, University of Baghdad, Baghdad, Iraq.

Article history: Received,25,February,2020, Accepted 23,June,2020, Published in April 2021

Doi: 10.30526/34.2.2611

\begin{abstract}
The attribute quality control charts are one of the main useful tools to use in control of quality product in companies. In this paper utilizing the statistical procedures to find the attribute quality control charts for through fuzzified the real data which we got it from Baghdad Soft Drink Company in Iraq, by using triangular membership function to obtain the fuzzy numbers then employing the proposed ranking function to transform to traditional sample. Then, compare between crisp and fuzzy attribute quality control.
\end{abstract}

Keywords: Quality Control, Fuzzy Set Theory, Attributes Control Charts, Ranking function.

\section{Introduction}

Statistical procedures are one of the main useful tools in supervision the production process by using control charts and sample inspection plans and these two procedures depend on the random variations which happen in terminology then determine the production process is subject to specifications of quality control or not, since the units produced differ in quality, if these imbalances and deviations are minor, the production is acceptable but if these differences and deviations exceed certain limits. The production is not acceptable.

Statistical methods were used in the field of quality control for the first time in 1924 by researcher (Shewart). The company makes the objective in manufacturing the items which vacant from defective and congruent to specifications, then utilizing the control charts to esteem the statistical techniques to control the quality production.

Control Chart is one of the most common statistical methods used in terms of monitoring the changes that occur during the stages of product process, it is determined by whether the process is statistically accurate by the observation recorded from the samples drawn.

The data was taken from one of the important production plants is a laboratory for the production of drinking water bottles to detect the specifications and efficiency of production 
accurately and quickly, the results showed that fuzzy control chart are more accurate and economically faster in controlling the quality of production, leading to the detection of defective units during the production process, which helps to detect error quickly.

Zadeh is the first from discover fuzzy set theory in (1965)[1]. Bradshaw (1983), for the first time used fuzzy sets as a basis for the explanation of the measurement of conformity of each product units with the specifications[2]. T. Raz and J. Wang (1990) have attempted to extend the use of control charts to allow for linguistic variables[3]. Ray Cheng et al (1995), proposed economic statistical np-control chart design[4]. F.Franceschine and D.Romano (1999), proposed a method for the online control of qualitative of the product/service using control charts for linguistic variables[5]. K.Latva-Kayra (2001), proposed EWMA and CUSUM with fuzzy control limits and their fuzzy combination is used[6]. M.Gulbayand C.Kahraman(2006), the direct fuzzy approach to fuzzy control charts without any distrotion, and fuzzy abnormal pattern rules based on the probabilities of fuzzy events is proposed[7]. Chih-Hsuan Wang-Way Kuo (2007), multiresolution relied on robust fuzzy clustering approach[8]. H.Moheb Alizadeh, A.R.Arshadi Khamseh and S.M.T Fatemi Ghomi (2010), developed multivariate variable control charts in fuzzy mode[9]. Osman Taylan, and Ibrahim A.Darrab (2012), describe the use of artificial intelligence (AI) methods such as fuzzy logic and neural networks in quality control and improvement[10]. Mohammad Hossein and IR (2014), provide a literature review of the control chart under a fuzzy environment with proposing several classifications and analyzes[11]. P.Fernández and other IR (2015), the use of fuzzy control charts becomes inevitable when statistical data considered are vague or affected by uncertainty[12]. M.Hadi and M.Mahmoudzadeh (2017), presented the fuzzy statistical process control development for attribute quality control chart by using Monte Carlo simulation method[13]. The aim of the study is applying the crisp control chart and fuzzy control chart for real data by utilizing of triangular membership function. Then employing the proposed ranking function to find the attribute quality control when $(\mathrm{w}=0.2$, $\lambda=0.5)$ and $(w=0.6, \lambda=0.9)$. This paper is organized as follows. In section 2 , showing the attribute control chart technique. In section 3, showing the fuzzy set theory. In section 4 , introducing the new method of ranking function. In section 5, introduction the application of real data. In section 6, numerical results are shown. In section 7, conclusions are given.

\section{Attributes Control Charts}

In some cases, production units can be divided into two types defective and invalid production units and non-defective and valid production units, this means that the units produced are described by specific properties or characteristics. If assume the withdrawal of $n_{i}$ from random samples of equal sizes from a specific production process during regular interval and which distributes Binomial Distribution and assume that the defective ratios in production are (p) and the non-defective ratios are (1-p) then the ratios of defective values are between the two limits $\left(\bar{p} \mp \sqrt{\frac{p(1-p)}{n}}\right)$ where as $\bar{p}=\frac{\sum p_{i}}{n}$ is the defective rate of proportions, the control chart is represented by three parallel lines:

The middle limit of attribute control is:

$\mathrm{CL}=\bar{P}=\frac{\sum p_{i}}{n}$

The upper limit of attribute control is: 
$\mathrm{UCL}=\left(\bar{p}+\sqrt{\frac{p(1-p)}{n}}\right)$

The lower limit of attribute control is:

$\mathrm{LCL}=\left(\bar{p}-\sqrt{\frac{p(1-p)}{n}}\right)$

Where the proportion of defective is:

$\mathrm{P}=\frac{\text { defective }}{\text { production }}$

If one or more defective proportions are outside the upper and lower control limits, then the production process is outside the control limit. Otherwise, the production process is under control.

3-Fuzzy set: [14] Let $X$ be the universal set. A fuzzy set in $X$ is a set of ordered pairs, $\mathrm{A}=\left\{\left(\mathrm{x}, \mu_{A}(x)\right) ; x \in X\right\}$, where $\mu_{A}: X \rightarrow[0,1]$ is called the membership set.

$\boldsymbol{\alpha}$-Cats of a Fuzzy Set:[14] The crisp set that contains all the elements of $X$ that have nonzero membership grades in a fuzzy set $A$ is called the support of the set $A$, denoted by $\operatorname{Supp}(\mathrm{A})$. i.e., $\operatorname{Supp}(\mathrm{A})=\{\mathrm{x} \in X: \mathrm{A}(\mathrm{x}) \geq 0\}$.

The membership function that we use it is as following

$\mu_{A}(x)=\left\{\begin{array}{cc}\frac{\lambda(x-a)}{(b-a} & a \leq x \leq b \\ \lambda & x=b \\ \frac{\lambda(c-x)}{(c-b)} & b \leq x \leq c\end{array}\right.$

4-Ranking function: [8] Let $\tilde{\mathrm{A}}, \tilde{B} \in \mathrm{E}$, define is ranking of $\mathrm{A}$, B by $\mathrm{R}($.$) on \mathrm{E}$, i.e

$$
\begin{aligned}
& \mathrm{R}(\tilde{\mathrm{A}})>\mathrm{R}(\tilde{B}) \leftrightarrow \tilde{\mathrm{A}}>\tilde{B} \\
& \mathrm{R}(\tilde{\mathrm{A}})<\mathrm{R}(\tilde{B}) \leftrightarrow \tilde{\mathrm{A}}<\tilde{B} \\
& \mathrm{R}(\tilde{\mathrm{A}})=\mathrm{R}(\tilde{B}) \leftrightarrow \tilde{\mathrm{A}} \approx \tilde{B}
\end{aligned}
$$

Considure that the triangular fuzzy numbers represented by $\tilde{A}=(a, b, c)$, where $b$ is the all sample, $\mathrm{a}$ is the lift width and $\mathrm{c}$ is the right width.

Now, presented the arbitrary fuzzy numbers $\tilde{\mathrm{A}}(\alpha)$ by an ordered pair of function $\left[\tilde{\mathrm{A}}^{L}(\alpha), \tilde{\mathrm{A}}^{U}(\alpha)\right]$, where $\tilde{\mathrm{A}}^{L}(\alpha)$ is a bounded left continuous nondecreasing function over $[0,1]$ and $\tilde{\mathrm{A}}^{U}(\alpha)$ is a bounded left continuous nonincreasing function over $[0,1], \tilde{\mathrm{A}}^{L}(\alpha) \leq \tilde{\mathrm{A}}^{U}(\alpha)$. Where $\tilde{\mathrm{A}}^{L}(\alpha)=\inf \{x \mid \tilde{\mathrm{A}}(x) \geq \alpha\}$ and $\tilde{\mathrm{A}}^{U}(\alpha)=\sup \{x \mid \tilde{\mathrm{A}}(x) \geq \alpha\}$.

Now, the utilizing the triangular membership function to find the new ranking function which is as follows:

$$
\begin{array}{ll}
\alpha=\frac{\lambda(x-a)}{(b-a)} & \alpha=\frac{\lambda(c-x)}{(c-b)} \\
x=\frac{\alpha}{\lambda}(b-a)+a & x=c-\frac{\alpha}{\lambda}((c-b) \\
\tilde{\mathrm{A}}^{L}(\alpha)=a+\frac{\alpha}{\lambda}(b-a) & \tilde{\mathrm{A}}^{u}(\alpha)=c-\frac{\alpha}{\lambda}(c-b) \\
\mathrm{R}(\tilde{\mathrm{A}})=\frac{\int_{w}^{\lambda}\left[\breve{A}^{L}(\alpha)+\breve{A}^{u}(\alpha)\right] d \alpha}{\int_{w}^{\lambda} \alpha d \alpha}=\frac{\int_{w}^{\lambda}\left[a+\frac{\alpha}{\lambda}(b-a)+c-\frac{\alpha}{\lambda}(c-b)\right] d \alpha}{\int_{w}^{\lambda} \alpha d \alpha} & \\
=\frac{\int_{w}^{\lambda}\left[(a+c)+\frac{\alpha}{\lambda}(2 b-a-c)\right] d \alpha}{\int_{w}^{\lambda} \alpha d \alpha}=\frac{\left.\left[(a+b) \alpha+\frac{\alpha^{2}}{2 \lambda}(2 b-a-c)\right]\right|_{w} ^{\lambda}}{\left.\frac{\alpha^{2}}{2}\right|_{w} ^{\lambda}} &
\end{array}
$$




$$
\begin{aligned}
& =\frac{(a+c)(\lambda-w)+\frac{\left(\lambda^{2}-w^{2}\right)}{2 \lambda}(2 b-a-c)}{\frac{\lambda^{2}-w^{2}}{2}}=\frac{(a+c)(\lambda-w)+\frac{(\lambda-w)(\lambda+w)}{2 \lambda}(2 b-a-c)}{\frac{(\lambda-w)(\lambda+w)}{2}} \\
& =\frac{2 \lambda(a+b)+(\lambda+w)(2 b-a-c)}{2 \lambda} * \frac{2}{(\lambda+w)}=\frac{2 \lambda(a+b)+(\lambda+w)(2 b-a-c)}{\lambda(\lambda+w)} \\
& =\frac{\lambda(2 b+a+c)+w(2 b-a-c)}{\lambda(\lambda+w)}
\end{aligned}
$$

\section{Application}

Baghdad Soft Drinks Company is one of the most important companies operating in the province of Baghdad /Zaafaraniya and the establishment of this company dates back to the 1960s as one of the establishments of the Ministry of Industry and Minerals where issued a founding license from the Directorate General of Industrial Development no. 474 in $1961 / 12 / 21$. However, it became a mixed company in 1989 in accordance with companies Law no. 36 of 1983 and procedures of establishing the company were completed by issuing the certificate of incorporation under the decision of the Registrar of companies in the Ministry of Commerce no. (m.s/3315) in 1989/3/23.

The company has 5 factories, each of which contains production lines:

a) Degla factory: consists of four production lines.

b) Euphrates factory: consists of four production lines.

c) Shatt al-Arab factory: consists of two production lines.

d) Rafidain factory: consist of one production line.

e) Aquafina factory: consists of two production lines.

In addition to three factories to manufacture carbon dioxide gas.

The company is licensed to produce soft drinks from Pepsi Co. International and latter takes samples from markets and is examined to assess the quality of production and the company is adjacent to distribute its products in central and southern Iraq.

\section{Numerical Results}

The samples that we get it from Baghdad Soft Drinks Company are as follows:

Table 1. contain defective and production from company

\begin{tabular}{|c|c|c|c|c|c|c|c|c|}
\hline $\mathrm{n}$ & defective & production & $\mathrm{n}$ & defective & production & $\mathrm{n}$ & defective & production \\
\hline 1 & 2613 & 1035510 & 11 & 3953 & 1205130 & 21 & 224 & 179970 \\
\hline 2 & 3337 & 1161630 & 12 & 910 & 809400 & 22 & 920 & 590268 \\
\hline 3 & 3087 & 1118460 & 13 & 1932 & 1064520 & 23 & 778 & 1007520 \\
\hline 4 & 2650 & 1104840 & 14 & 452 & 366990 & 24 & 512 & 406200 \\
\hline 5 & 1974 & 896250 & 15 & 947 & 654330 & 25 & 263 & 528000 \\
\hline 6 & 2566 & 1150950 & 16 & 3544 & 1115640 & 26 & 896 & 1204500 \\
\hline 7 & 3417 & 1138590 & 17 & 1632 & 1120320 & 27 & 731 & 749592 \\
\hline 8 & 1405 & 772566 & 18 & 845 & 769740 & 28 & 444 & 808500 \\
\hline 9 & 1837 & 982566 & 19 & 1562 & 1054260 & 29 & 2054 & 1118700 \\
\hline 10 & 2539 & 1121220 & 20 & 1602 & 1060890 & 30 & 312 & 314130 \\
\hline
\end{tabular}

Now, applying attributes control charts in all samples of Table (1)

First, find (p) in equation (4).

$P_{1}=\frac{2613}{1035510}=0.002523$ and so that 
Ibn Al-Haitham Jour. for Pure \& Appl. Sci. 34 (2) 2021

Table 2. for the value $(\mathrm{P})$

\begin{tabular}{|c|l|l|l|l|l|}
\hline $\mathrm{n}$ & \multicolumn{1}{|c|}{$\mathrm{p}$} & $\mathrm{n}$ & \multicolumn{1}{c|}{$\mathrm{p}$} & $\mathrm{n}$ & \multicolumn{1}{c|}{$\mathrm{p}$} \\
\hline 1 & 0.002523394 & 11 & 0.003280144 & 21 & 0.001244652 \\
\hline 2 & 0.002872688 & 12 & 0.00112429 & 22 & 0.001558614 \\
\hline 3 & 0.002760045 & 13 & 0.001814902 & 23 & 0.000772193 \\
\hline 4 & 0.002398537 & 14 & 0.001231641 & 24 & 0.001260463 \\
\hline 5 & 0.00220251 & 15 & 0.001447282 & 25 & 0.000498106 \\
\hline 6 & 0.002229463 & 16 & 0.003176652 & 26 & 0.000743877 \\
\hline 7 & 0.00300108 & 17 & 0.001456727 & 27 & 0.000975197 \\
\hline 8 & 0.001818615 & 18 & 0.001097773 & 28 & 0.000549165 \\
\hline 9 & 0.001869595 & 19 & 0.001481608 & 29 & 0.00183606 \\
\hline 10 & 0.002264498 & 20 & 0.001510053 & 30 & 0.000993219 \\
\hline
\end{tabular}

Second compute the middle limit of attribute control in equation (1)

$\mathrm{CL}=\bar{P}=\frac{\sum_{1}^{30} P_{i}}{30}=\frac{0.051993}{30}=0.001733$

*After that, compute the upper limit of attribute control in equation (2)

$\mathrm{UCL}=\bar{P}+3 \sqrt{\frac{\bar{p}(1-\bar{p})}{n}}=0.024515$

Now, compute the lower limit of attribute control in equation (3)

$\mathrm{LCL}=\bar{P}-3 * \sqrt{\frac{\bar{p}(1-\bar{p})}{n}}=-0.02105$

Finally, drawing the charts of quality control with depend on the attributes control charts

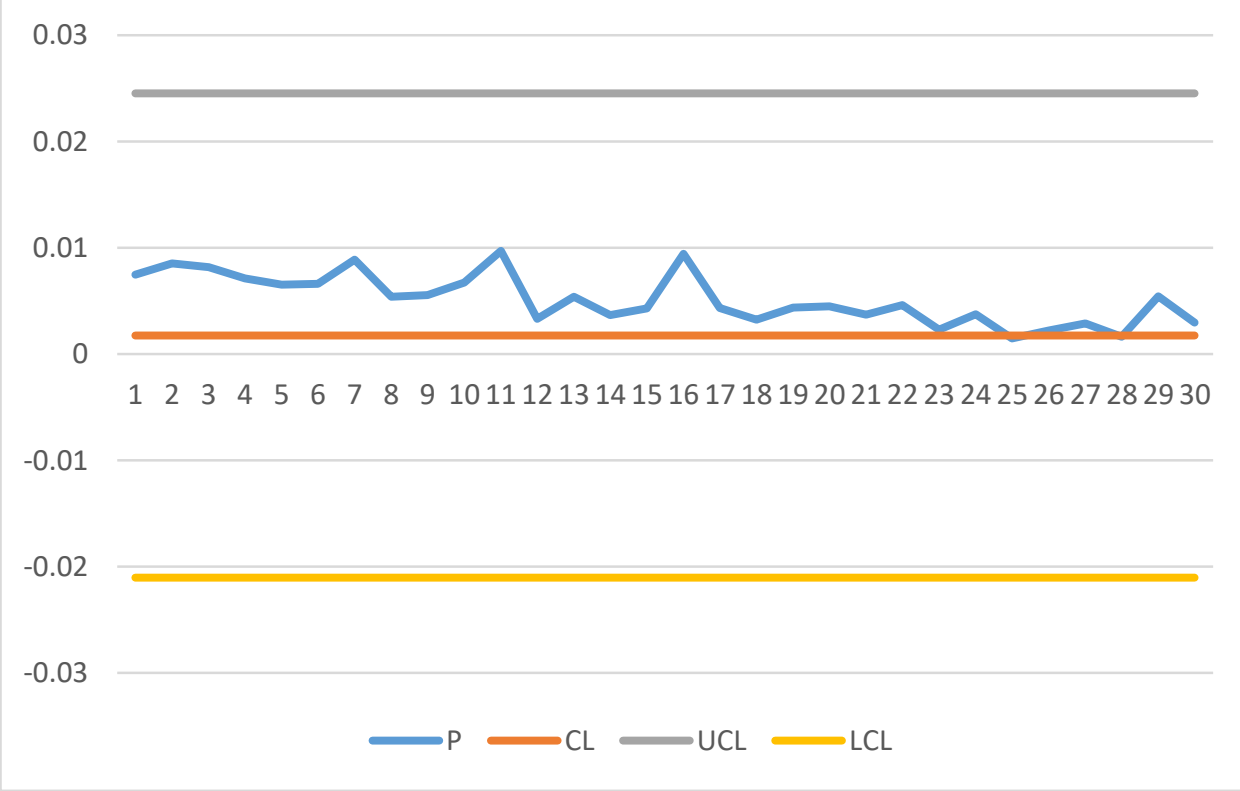

Figure 1. Contain the charts to the original values 
Then the fuzzified the data to make it vague numbers by using the membership function in equation (5) which are as follows:

$(a, b, c)=($ all samples $-\bar{x}$, all samples, all samples $+\bar{x})$

Therefore, applying the new ranking function in equation (7) to transform the fuzzy numbers to crisp numbers but the new ranking function depend upon $w, \lambda \in[0,1]$.

Now, computing a new ranking function by utilizing the values of $\mathrm{w}, \lambda$.

The values of the $\mathrm{w}, \lambda$ are $\lambda=0.5, \mathrm{w}=0.2$.

Then using equation (6) to find the ranking function

Table 3. Fuzzy ranking function of defective samples

\begin{tabular}{|c|l|l|l|l|l|}
\hline $\mathrm{n}$ & \multicolumn{1}{|c|}{ defective } & $\mathrm{n}$ & defective & $\mathrm{n}$ & defective \\
\hline 1 & 14931.42857 & 11 & 22588.57143 & 21 & 1280 \\
\hline 2 & 19068.57143 & 12 & 5200 & 22 & 5257.142857 \\
\hline 3 & 17640 & 13 & 11040 & 23 & 4445.714286 \\
\hline 4 & 15142.85714 & 14 & 2582.857143 & 24 & 2925.714286 \\
\hline 5 & 11280 & 15 & 5411.428571 & 25 & 1502.857143 \\
\hline 6 & 14662.85714 & 16 & 20251.42857 & 26 & 5120 \\
\hline 7 & 19525.71429 & 17 & 9325.714286 & 27 & 4177.142857 \\
\hline 8 & 8028.571429 & 18 & 4828.571429 & 28 & 2537.142857 \\
\hline 9 & 10497.14286 & 19 & 8925.714286 & 29 & 11737.14286 \\
\hline 10 & 14508.57143 & 20 & 9154.285714 & 30 & 1782.857143 \\
\hline
\end{tabular}

Now, applying attributes control charts in all samples when $\lambda=0.5, \mathrm{w}=0.2$

First, find (p) in equation (4).

$P_{1}=\frac{14931.42857}{1035510}=0.014419396$ and so that

Table 4. For the value $(\mathrm{P})$ when $\lambda=0.5, \mathrm{w}=0.2$

\begin{tabular}{|c|l|l|l|l|l|}
\hline $\mathrm{n}$ & \multicolumn{1}{|c|}{$\mathrm{p}$} & $\mathrm{n}$ & \multicolumn{1}{|c|}{$\mathrm{p}$} & $\mathrm{n}$ & \multicolumn{1}{c|}{$\mathrm{p}$} \\
\hline 1 & 0.014419396 & 11 & 0.01874368 & 21 & 0.007112296 \\
\hline 2 & 0.016415357 & 12 & 0.006424512 & 22 & 0.008906366 \\
\hline 3 & 0.015771686 & 13 & 0.010370871 & 23 & 0.004412532 \\
\hline 4 & 0.013705928 & 14 & 0.00703795 & 24 & 0.007202645 \\
\hline 5 & 0.012585774 & 15 & 0.008270183 & 25 & 0.00284632 \\
\hline 6 & 0.012739786 & 16 & 0.018152297 & 26 & 0.004250726 \\
\hline 7 & 0.01714903 & 17 & 0.008324152 & 27 & 0.005572555 \\
\hline 8 & 0.010392085 & 18 & 0.00627299 & 28 & 0.003138086 \\
\hline 9 & 0.010683397 & 19 & 0.008466331 & 29 & 0.01049177 \\
\hline 10 & 0.012939986 & 20 & 0.008628874 & 30 & 0.005675539 \\
\hline
\end{tabular}

Second compute the middle limit of attribute control in equation (1) 
$\mathrm{CL}=\bar{P}=\frac{\sum_{1}^{30} P_{i}}{30}=0.009903$

After that, compute the upper limit of attribute control in equation (2)

$\mathrm{UCL}=\bar{P}+3 * \sqrt{\frac{\bar{p}(1-\bar{p})}{n}}=0.06414$

Now, compute the lower limit of attribute control in equation (3)

$\mathrm{LCL}=\bar{P}-3 * \sqrt{\frac{\bar{p}(1-\bar{p})}{n}}=-0.04433$

Finally, drawing the charts of quality control with depend on the attributes control charts

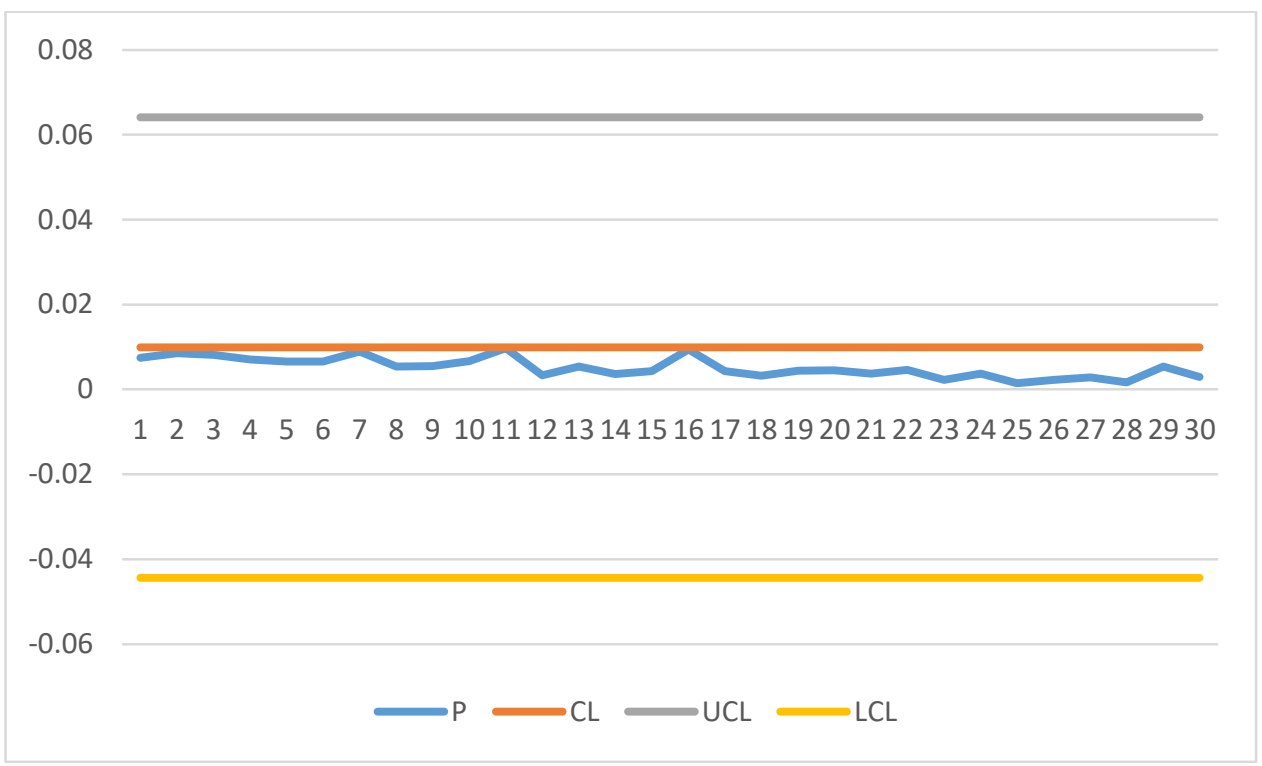

Figure 2. Contain the charts when $\lambda=0.5, \mathrm{w}=0.3$

Now, computing a new ranking function by utilizing the values of $w, \lambda$.

The values of the $\mathrm{w}, \lambda$ are $\lambda=0.9, \mathrm{w}=0.6$.

Then using equation (6) to find the ranking function

Table 5. Fuzzy ranking function of defective samples

\begin{tabular}{|l|l|c|l|l|l|}
\hline $\mathrm{n}$ & \multicolumn{1}{|c|}{ defective } & $\mathrm{n}$ & Defective & $\mathrm{n}$ & defective \\
\hline 1 & 6968 & 11 & 10541.33333 & 21 & 597.3333333 \\
\hline 2 & 8898.666667 & 12 & 2426.666667 & 22 & 2453.333333 \\
\hline 3 & 8232 & 13 & 5152 & 23 & 2074.666667 \\
\hline 4 & 7066.666667 & 14 & 1205.333333 & 24 & 1365.333333 \\
\hline 5 & 5264 & 15 & 2525.333333 & 25 & 701.3333333 \\
\hline 6 & 6842.666667 & 16 & 9450.666667 & 26 & 2389.333333 \\
\hline 7 & 9112 & 17 & 4352 & 27 & 1949.333333 \\
\hline 8 & 3746.666667 & 18 & 2253.333333 & 28 & 1184 \\
\hline 9 & 4898.666667 & 19 & 4165.333333 & 29 & 5477.333333 \\
\hline 10 & 6770.666667 & 20 & 4272 & 30 & 832 \\
\hline
\end{tabular}

Now, applying attributes control charts in all samples when $\lambda=0.9, \mathrm{w}=0.6$ 
First, find (p) in equation (4).

$P_{1}=\frac{6968}{1035510}=0.006729051$ and so that

Table 6. For the value $(\mathrm{P})$ when $\lambda=0.5, \mathrm{w}=0.2$

\begin{tabular}{|l|l|l|l|l|l|}
\hline $\mathrm{n}$ & \multicolumn{1}{|c|}{$\mathrm{p}$} & $\mathrm{n}$ & $\mathrm{P}$ & $\mathrm{n}$ & \multicolumn{1}{|c|}{$\mathrm{p}$} \\
\hline 1 & 0.006729051 & 11 & 0.008747051 & 21 & 0.003319072 \\
\hline 2 & 0.0076605 & 12 & 0.002998106 & 22 & 0.004156304 \\
\hline 3 & 0.00736012 & 13 & 0.00483974 & 23 & 0.002059182 \\
\hline 4 & 0.0063961 & 14 & 0.003284377 & 24 & 0.003361234 \\
\hline 5 & 0.005873361 & 15 & 0.003859419 & 25 & 0.001328283 \\
\hline 6 & 0.005945234 & 16 & 0.008471072 & 26 & 0.001983672 \\
\hline 7 & 0.008002881 & 17 & 0.003884604 & 27 & 0.002600526 \\
\hline 8 & 0.00484964 & 18 & 0.002927395 & 28 & 0.00146444 \\
\hline 9 & 0.004985585 & 19 & 0.003950955 & 29 & 0.004896159 \\
\hline 10 & 0.00603866 & 20 & 0.004026808 & 30 & 0.002648585 \\
\hline
\end{tabular}

Second compute the middle limit of attribute control in equation (1)

$\mathrm{CL}=\bar{P}=\frac{\sum_{1}^{30} P_{i}}{30}=0.004621604$

After that, compute the upper limit of attribute control in equation (2)

$\mathrm{UCL}=\bar{P}+3 * \sqrt{\frac{\bar{p}(1-\bar{p})}{n}}=0.041770943$

Now, compute the lower limit of attribute control in equation (3)

$\mathrm{LCL}=\bar{P}-3 * \sqrt{\frac{\bar{p}(1-\bar{p})}{n}}=-0.032527735$

Finally, drawing the charts of quality control with depend on the attributes control charts

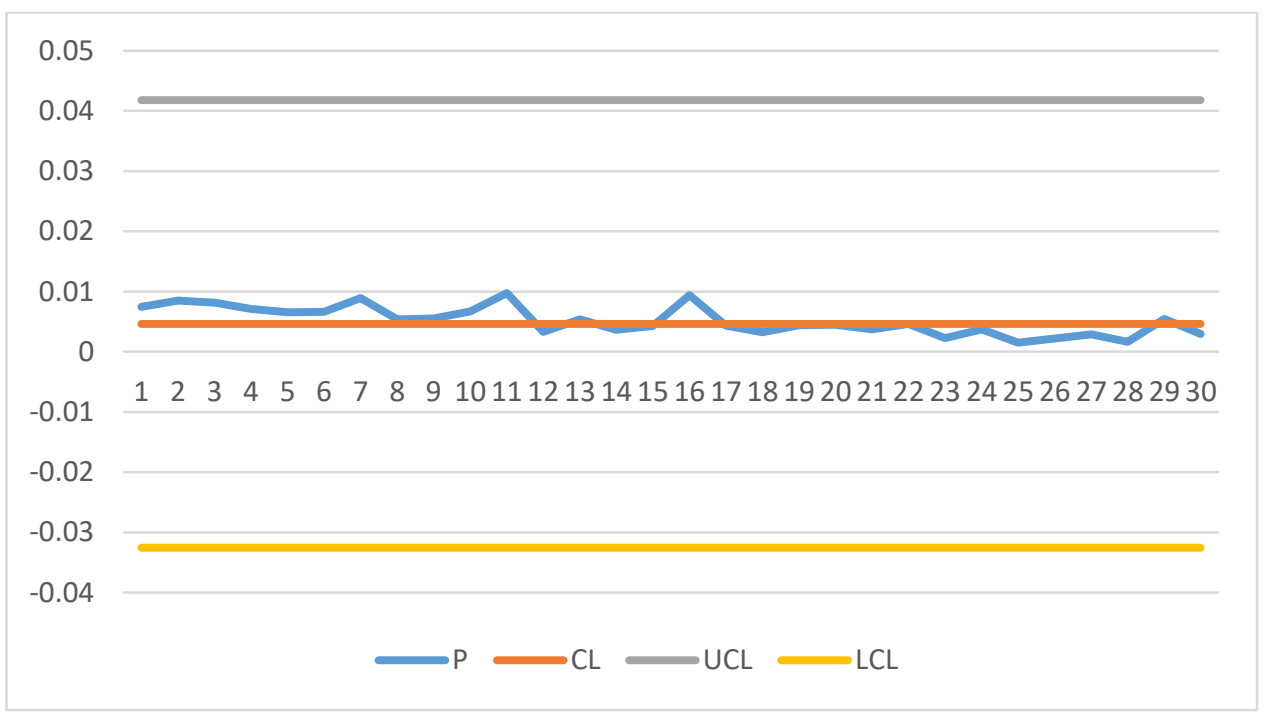

Figure 3. Contain the charts when $\lambda=0.9, \mathrm{w}=0.6$ 


\section{Conclusion}

In beginning of employment the attribute quality control chart to calculate the proportion defective for all samples. Applying the triangular membership function to get the fuzzy numbers of defective for all samples. Then carrying out the proposed ranking function twice, the first through using ( $\mathrm{w}=0.2, \lambda=0.5)$, the second through using ( $\mathrm{w}=0.6, \lambda=0.9)$ to obtain the fuzzy number for all samples. After that, carrying out the fuzzy quality control to compute the proportion defective for all samples. Finally, comparing between crisp and fuzzy control charts for all samples of production are under control limits.

\section{References}

1.Zadeh, L.A., Fuzzy sets, Information and control. 1965, 8, 3, 338-353.

2.Bradshaw, Jr, C.W., A fuzzy set theoretic interpretation of economic control limits. European Journal of Operational Research. 1983, 13, 403-408.

3.WANG, J.-H. and T. RAZ, On the construction of control charts using linguistic variables. The International Journal of Production Research, 1990. 28,3,477-487.

4.Wang, R.C. ; Chen,C.H. Economic statistical np-control chart designs based on fuzzy optimization. International Journal of Quality \& Reliability Management, 1995.

5.Franceschini, F. ; Romano, D. Control chart for linguistic variables: a method based on the use of linguistic quantifiers. International Journal of Production Research, 1999,37, 16, 37913801 .

6.Latva-Käyrä, K., Fuzzy logic and SPC,in Industrial Applications of Soft Computing., Springer. 2001, 197-210.

7.Gülbay, M. ; Kahraman, C. Development of fuzzy process control charts and fuzzy unnatural pattern analyses. Computational statistics \& data analysis, 2006,51,1,434-451.

8.Hajjari, T. ; Barkhordary, M. Ranking fuzzy numbers by sign length.in 7th Iranian Conference on Fuzzy Systems, Iran. 2007.

9.MOHEB, A.H., ARSHADI, K.A. ; FATEMI, G.S. Fuzzy development of multivariate variable control charts using the fuzzy likelihood ratio test. 2010.

10.Taylan, O. ; Darrab, I.A.Fuzzy control charts for process quality improvement and product assessment in tip shear carpet industry. Journal of Manufacturing Technology Management, 2012.

11.Sabegh, M.H.Z., et al., A literature review on the fuzzy control chart; classifications \& analysis. International Journal of Supply and Operations Management, 2014,1,2 ,167.

12.Pastuizaca Fernández, M.N., A. Carrión García, and O. Ruiz Barzola, Multivariate multinomial T 2 control chart using fuzzy approach. International Journal of Production Research, 2015, 53,7, 2225-2238.

13.Madadi, M. ; Mahmoudzadeh, M. A fuzzy development for attribute control chart with Monte Carlo simulation method. Management Science Letters, 2017, 7, 11,555-564.

14.George, M., Fuzzy Mathmatics_Application in Economic, Campus Books International. 2008, 555-564. 